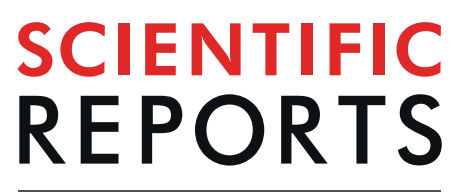

natureresearch

\title{
Orthogonal Representations of Object Shape and Category in Deep Convolutional Neural Networks and Human Visual Cortex
}

\begin{abstract}
Astrid A. Zeman ${ }^{1 *}$, J. Brendan Ritchie ${ }^{1}{ }^{1}$, Stefania Bracci ${ }^{1,2}$ \& Hans Op de Beeck ${ }^{1}$
Deep Convolutional Neural Networks (CNNs) are gaining traction as the benchmark model of visual object recognition, with performance now surpassing humans. While CNNs can accurately assign one image to potentially thousands of categories, network performance could be the result of layers that are tuned to represent the visual shape of objects, rather than object category, since both are often confounded in natural images. Using two stimulus sets that explicitly dissociate shape from category, we correlate these two types of information with each layer of multiple CNNs. We also compare CNN output with $\mathrm{fMRI}$ activation along the human visual ventral stream by correlating artificial with neural representations. We find that CNNs encode category information independently from shape, peaking at the final fully connected layer in all tested CNN architectures. Comparing CNNs with fMRI brain data, early visual cortex (V1) and early layers of CNNs encode shape information. Anterior ventral temporal cortex encodes category information, which correlates best with the final layer of CNNs. The interaction between shape and category that is found along the human visual ventral pathway is echoed in multiple deep networks. Our results suggest CNNs represent category information independently from shape, much like the human visual system.
\end{abstract}

In recent years, the performance of Deep Convolutional Neural Networks (CNNs) has improved significantly, such that they are able to meet ${ }^{1-3}$, and even surpass ${ }^{4}$ human performance in classifying objects. In light of these impressive findings, these artificial networks are increasingly compared to their biological counterparts, resulting in an accumulation of evidence for their use as a benchmark model of visual object recognition ${ }^{5,6}$. For example, the internal representations of CNNs show correspondence with human ventral temporal cortex (VTC) as measured by fMRI, as well as with primate inferotemporal cortex (IT) measured using single cell recordings ${ }^{7-12}$. The correspondence between deep networks and neural representations along the visual pathway has even allowed for accurate neural response prediction of single-cell recordings in $\mathrm{IT}^{9}$ as well as $\mathrm{fMRI}^{13}$. Representational similarities have been further extended from the spatial into the temporal domain, with results showing a corresponding ordering of processing between CNNs and the human visual brain using MEG $^{14}$. These accumulating findings showcase the ability of CNNs to model neurons from single unit responses to entire populations, spanning the multiple scales and dimensions used to study neural activity, and make CNNs some of the best models to date for studying vision in the human and primate brain.

While these feats are impressive, it is unclear to what extent these results are easily interpretable in terms of categorical representations. Object category information can often be confounded with low-level visual features, such as colour, texture, and shape ${ }^{15}$. In this paper, we highlight the significant interaction between shape and category that is known to occur in natural images ${ }^{16}$ and address the possibility that these networks may distinguish between object categories by relying upon visual features, such as shape, rather than high-level category representations. Indeed, the shape similarity of objects has already been capitalised on in the machine learning field to improve performance ${ }^{17}$. CNNs are proficient at representing the perceived shape of objects, as opposed to their physical shape ${ }^{18}$ and it has been claimed that CNNs rely heavily upon local shape information for classification ${ }^{19}$. Two-dimensional regular vs irregular shape representations have been found in monkey IT, which are highly comparable to late layers of $\mathrm{CNNs}^{12}$. Furthermore, CNNs mimic a behavioural bias in humans known as 
the "shape-bias", which is the preference to categorise an object based on shape rather than colour ${ }^{20}$. In contrast to humans, CNNs rely more heavily upon local shape information for classification, known as texture-bias, which may potentially cause a greater discrepancy in performance than shape-bias ${ }^{21}$. Given that these networks are adept at representing object shape, to a degree that may even be greater than humans, it is possible they are taking advantage of shape-based features, instead of category information, to classify object images.

Recent neuroimaging studies have begun to de-confound category from visual features, including shape, in order to investigate their interaction along the visual ventral pathway ${ }^{10,16,22,23}$. VTC in humans is one of the main category-selective areas $^{24}$, distinguishing, for example, between animate and inanimate objects ${ }^{25,26}$. To build up this category-related representation, visual information is processed in a series of stages along the ventral visual pathway, from primary visual cortex (area V1) through to $\mathrm{VTC}^{24}$. In recent years, the exact role of VTC has come under question, in particular whether this area encodes category-specific information, or simply the low-level visual properties associated with category, such as colour, shape, size and texture ${ }^{15,27,28}$. Proklova, Kaiser \& Peelen $^{23}$ found that VTC encodes texture and outline alongside category-specific information that is not present in earlier visual areas. Another higher visual area, lateral occipitotemporal complex (LOTC), was found to encode category-associated shape properties as well as category-selective information ${ }^{22}$. Other category-orthogonal object properties, including size, position and pose, show higher population decoding performance in monkey IT (analogous to human VTC) compared to early visual areas, contrary to what was previously believed ${ }^{10}$. Indeed, the majority of visual object representations in IT may be accounted for by object shape, or other low-level visual properties, rather than category ${ }^{29}$. Nevertheless, studies that explicitly de-confound category from more low-level properties suggest that the category selectivity cannot be fully explained by these other properties ${ }^{10,16,22}$, and point towards a so-called "feature-dependent categorical code ${ }^{15}$." Such a code stands in contrast to a more abstract categorical code, and includes clear selectivity for the features that define and correlate with the coded category (see Bracci, Ritchie \& Op de Beeck ${ }^{15}$ for more information).

In this paper, we explicitly dissociate shape from category in two stimulus sets to determine: (i) how CNNs represent object shape and category when they are independent from one another; and (ii) how these artificial representations correspond with shape and category representations in human visual cortex. Using two carefully designed stimulus sets, which orthogonalise shape and category, we assess four top-performing CNNs in their ability to represent category independently from shape layer by layer. Taking the same two stimulus sets, we measure human $\mathrm{fMRI}$ responses when viewing these images and assess the interaction between shape and category along the visual ventral stream. Finally, we compare artificial representations with human fMRI responses for the same two stimulus sets, to evaluate how closely CNNs reflect biological representations.

\section{Methods}

We aimed to determine the relationship between models of shape and category, CNNs, and neural responses in the human visual ventral pathway. We tested object shape and category representation in four top-performing CNNs and compared this with behavioural ratings of shape and category as well as human fMRI response patterns from experiments in two previous studies ${ }^{16,30}$. Human fMRI responses that are reported here use different ROIs than in the original studies. Some findings related to the second stimulus set have been published in previous work, which we acknowledge here and within the paper. For the second stimulus set, human behavioural ratings were compared to three of four of the CNNs we present here, in Kubilius et al. ${ }^{18}$. Human fMRI responses to shape and category were previously reported in Bracci \& Op de Beeck ${ }^{16}$.

Below we describe participants, stimulus sets, $\mathrm{CNN}$ architectures, the neuroimaging experiments, and data analysis.

Participants. All participants gave written informed consent. All experiments were approved by the Ethics Committee at KU Leuven and the University Hospitals Leuven. All methods were performed in accordance with the relevant guidelines and regulations. For the behavioural ratings, each stimulus set was rated by an independent group of participants ( $\mathrm{N}=4$ for set $\mathrm{A} ; \mathrm{N}=16$ for set $\mathrm{B}$ ). For the neuroimaging experiments, there were 15 participants ( 8 females, mean age of 30 years) scanned in $\mathrm{fMRI}$ experiment $\mathrm{A}$, none whom were excluded. There were also 15 participants ( 8 females, mean age of 24 years) scanned for fMRI experiment B, with one subject who was excluded due to excessive head motion. All subjects had normal or corrected vision.

Stimulus sets. The stimuli in both experiments were designed to dissociate shape from category information. Both stimulus sets are grayscale images of objects on a white or grey background, centred at the origin and presented at a normal viewing angle (see Fig. 1). Set A contains 32 unique images, divided into 2 equally sized categories (animal vs non-animal) and 2 equally sized groups of shapes (low and high aspect ratio). Set B contains 54 images divided into 6 object categories (minerals, animals, fruit/veg, music, sport and tools) and 9 shape types. The model design for each stimulus set, which orthogonalises shape from category, is illustrated in Fig. 1. For additional information about the stimulus sets, refer to Ritchie and Op de Beeck ${ }^{30}$ and Bracci and Op de Beeck ${ }^{16}$, for Set A and B respectively.

To confirm that shape was not predictive of category information for each of the stimulus sets, we analysed the images using low-level GIST descriptors ${ }^{31}$ and tested how well these visual features predicted shape or category using Linear Discriminant Analysis (LDA). GIST provides a low dimensional representation of an image based on spectral and coarsely localised information. We defined the GIST descriptors to include 8 orientations over 8 scales and combined this with LDA. For Set A, we ran a two-way classification using a leave-one-level out procedure, for example, training on bar stimuli and generalising to blob stimuli to test for animacy classification. For Set B, we followed a six-way classification scheme using a leave-one-level out test procedure, permuting across all possible groups of train and test combinations and averaging across results. For example, we selected six shape clusters of the total nine, trained an LDA on GIST descriptors from five clusters $(5 \times 6=30$ images $)$ and tested 
A

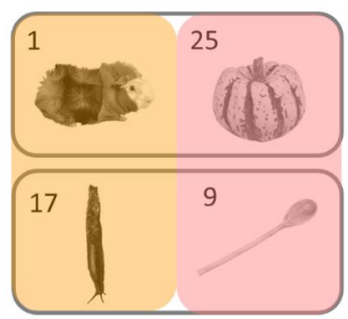

category

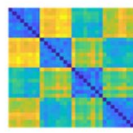

shape

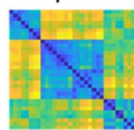

B

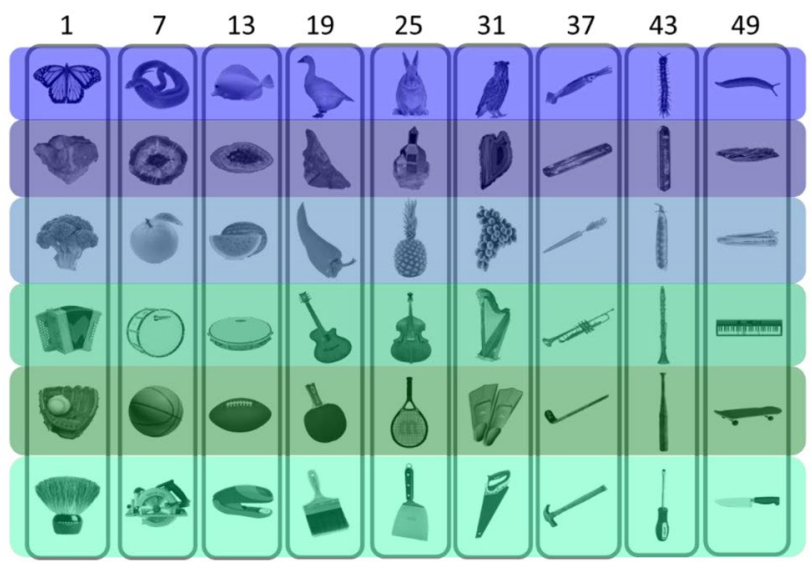

category

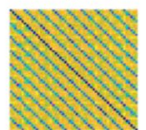

shape

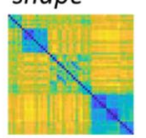

Figure 1. (A) 32 stimuli in 2 categories (animal and non-animal), (B) 54 stimuli in 6 categories (animals, minerals, fruit/vegetables, music, sports equipment, tools). Left: Each category division is highlighted by a distinct colour. Common shape information is circled in grey. Numbers indicate indexing for RDMs. Due to copyright restrictions, not all images are shown in Set A and the ones displayed are representative. Set A images are published in compliance with a CC BY-SA license (https://creativecommons.org/licenses/by-sa/3.0/) and their sources are: guinea pig (https://commons.wikimedia.org/wiki/File:AniarasKelpoKalle.jpg by Tavu); squash (https://commons.wikimedia.org/wiki/File:Festival-Squash.jpg by Evan-Amos); slug (Black Slug at Aggregate Ponds, https://www.flickr.com/photos/brewbooks/2606728819 by brewbooks); and wooden spoon (https:// upload.wikimedia.org/wikipedia/commons/7/7b/Wooden_Spoon.jpg by Donovan Govan). Images have been changed to greyscale and have the background removed. The final two images have also been rotated. Set B images are published in compliance with a CC-BY license (https://creativecommons.org/licenses/by/4.0/) and are re-used from Fig. $5 \mathrm{a}$ in Kubilius, Bracci and Op de Beeck ${ }^{18}$. Right: Shape and category RDMs. These behavioural models are obtained via multiple object arrangement ${ }^{32}$; see methods.

whether the algorithm could predict the 6 different categories from the held out images. All six-way shape and category combinations were tested (totalling 504 possible outcomes), which were averaged across cross-validation folds to provide a summary result.

Behavioural ratings. Each stimulus set was rated on object category and shape properties by means of the multiple object arrangement method ${ }^{32}$. Participants rated similarity in two task contexts: for object category, "arrange the images based on the semantic similarity among objects"; for object shape, "arrange the images based on perceived object shape similarity". These models, based on behavioural data, represent the stimulus psychological space and are shown in Fig. 1A,B.

fMRI experiments. Here we provide a summary of the fMRI procedures and analyses, the full details are provided in Ritchie and Op de Beeck ${ }^{30}$ for experiments using Set A and Bracci and Op de Beeck ${ }^{16}$ for Set B.

Preprocessing and analysis. All imaging data was pre-processed and analysed using SPM and MATLAB. For each participant, fMRI data was slice-time corrected, motion corrected (using spatial realignment to the first image), coregistered to each individual's anatomical scan, segmented and spatially normalised to the standard MNI template. Functional images were resampled to $3 \times 3 \times 3 \mathrm{~mm}$ voxel size and spatially smoothed by convolving with a Gaussian kernel of $6 \mathrm{~mm}$ FWHM for Set A and $4 \mathrm{~mm}$ FWHM for Set B ${ }^{33}$. After pre-processing, a GLM was used to model the BOLD signal for each participant, for each stimulus, at each voxel. Regressors for the GLM included each stimulus condition of interest (32 for A, 54 for B) and 6 motion correction parameters ( $x, y$ and $\mathrm{z}$ coordinates for translation and rotation). Each predictor had its time course modelled as a boxcar function convolved with the canonical haemodynamic response function, producing a single estimate for each voxel per predictor for every run. The beta weights fitted to each GLM were used to create Representational Dissimilarity Matrices (RDMs) for each participant (defined below). 
Regions of interest (ROIs). Neural representational content was investigated in three main ROIs in visual cortex: primary visual cortex (V1), and ventral temporal cortex (VTC), which was split into posterior (VTC post) and anterior (VTC ant) halves. These ROIs were chosen for their relevance in both object shape and category information processing ${ }^{24}$. VTC is bounded laterally by the occipitotemporal sulcus (OTS), posteriorly by the posterior transverse collateral sulcus ( $\mathrm{ptCoS}$ ) and anteriorly by the anterior tip of the mid-fusiform sulcus (MFS) ${ }^{24}$. ROIs were defined at the group level by combining the anatomical criteria above (using the Neuromorphometrics atlas in SPM) with functional criteria (all active voxels for the contrast of all conditions versus baseline that responded to visual information exceeding the statistically uncorrected threshold of $p<0.001$ in a second-level analysis). For further details on ROI definition, please refer to Bracci, Kalfas \& Op de Beeck ${ }^{34}$ where the exact same ROI criteria were applied. We used a two-factor repeated-measures Analysis of Variance Model (ANOVA) to assess the interaction between two within-participant factors: conditions (shape, category) and area (V1, VTC post and VTC ant).

Deep neural network architectures. Each architecture consists of multiple convolutional layers followed by pooling operations and fully-connected layers. For each CNN, which was pre-trained on the ImageNet data$\mathrm{set}^{35}$, we ran a forward pass of each image in the stimulus set through the network. We output the activation of weights in each layer, resulting in a matrix with size of the nodes per layer times the stimulus set (32 for A, 54 for B). We calculated 1 - correlation for each activation pattern of one stimulus with another to obtain an RDM with size $\mathrm{N} \times \mathrm{N}$, where $\mathrm{N}=$ the number of stimulus conditions $(32 \times 32$ for $\mathrm{A}, 54 \times 54$ for $\mathrm{B})$. We did not include final softmax classification layers in our analysis, since we were interested in the structure of layer representations and not classification performance per se.

CaffeNet. CaffeNet is an implementation of AlexNet $^{1}$ in the Caffe deep learning framework ${ }^{36}$. CaffeNet is an 8-layer convolutional neural network (CNNs) with five convolutional layers and three fully connected layers.

VGG-19. VGG-193 was the top ranking CNN for single object localisation in ILSVRC 2014, and second-running in image classification ${ }^{35}$. VGG-19 consists of 19 weighted layers with an additional softmax read-out layer for classification. The architecture contains 16 convolutional layers separated by five max pooling layers, with the final 3 layers being fully-connected.

GoogLeNet. GoogLeNet ${ }^{2}$, also known as InceptionNet, was the top-performing architecture for image classification in ILSVRC 2014 35 . GoogLeNet is a 22-layer deep network, when counting only parameterised layers, or 27 layers deep if including pooling operations. All convolution, reduction and projection layers use rectified linear activation. The bottom layers of the network follow conventional convolutional neural network architecture, consisting of chained convolutional operations followed by max pooling. The top layers of the network replace multiple fully-connected layers with an average pooling layer, a single fully connected layer and a classification layer. The middle layers of the network differ substantially from traditional convolutional neural network structure, consisting of stacked "inception" modules, which are miniature networks containing one max pooling and 3 multi-sized convolution operations $(1 \times 1,3 \times 3$ and $5 \times 5$ convolutions $)$ in parallel configuration. Convolution operations inside inception modules are optimised with dimensionality reduction, by preceding expensive $3 \times 3$ and $5 \times 5$ convolution operations with $1 \times 1$ convolutions. Inception modules allow for increased width of the network, as well as depth, while maintaining a constant computational budget.

ResNet50. ResNets are a family of extremely deep architectures that won the ILSVRC classification task in $2015^{37}$. ResNet50 contains 50 stacked "residual units", which use a split-transform-merge strategy to perform identity mappings in parallel to $3 \times 3$ convolutions with rectification. ResNets, like GoogLeNet ${ }^{2}$, are multi-branch architectures, containing only 2 branches (performing identity projection and $3 \times 3$ convolutions) instead of GoogLeNet's maximum 4 branch inception modules (performing multi-size convolutions). Identity mappings perform a key role in the architecture's success, forcing the network to preserve features, rather than learn entirely new representations at every layer, as is the case with conventional $\mathrm{CNNs}^{38}$. The final 3 layers of ResNet50 are identical in design to GoogleNet, performing average pooling, transformation to 1000 dimensions using full connections and softmax classification (not included in our analysis).

Representational similarity analysis. We used Representational Similarity Analysis (RSA) to quantitatively compare CNN representations per layer with design models, behavioural ratings, and with fMRI neuroimaging data. RSA compares RDMs, which characterise the representational information in a brain or model ${ }^{39}$. Given a set of activity patterns (biological, behavioural or artificial) for a set of experimental conditions, the dissimilarity between patterns is computed as 1 minus the correlation across the units that compose the patterns. RDMs are symmetrical about a zero diagonal, where 0 denotes perfect correlation. RSA assesses second-order isomorphism, which is the shared similarity in structure between dissimilarity matrices ${ }^{40}$. Spearman rank order correlation was used to compare dissimilarity matrices, since the relationship between RDMs cannot be assumed to be linear ${ }^{39}$. In cases where there was any dependency relationship between shape and category RDMs (visible in the Set A behavioural data), we used partial correlation.

\section{Results}

Behavioural data. For each stimulus set, participants provided similarity judgments for the shape and category dimension (see Fig. 1, right column). For Set A, we found a significant correlation between the behavioural models for shape and category (Spearman's $\rho=0.4753, p<0.001$ permutation test with 1000 randomisations of stimulus labels) and so partial correlations were performed when carrying out RSA with Set A models (this 
includes comparisons with CNN layers and with brain data). For Set B, we found no significant correlation between behavioural models for shape and category $(\rho=0.006, p=0.8209)$. Behavioural ratings for Set B have been previously used in Bracci \& Op de Beeck ${ }^{16}$.

Low-level shape analysis of stimuli. Using GIST ${ }^{31}$ descriptors of each image and combining this with LDA, we confirmed that category could not be predicted based upon these low-level descriptors whereas shape could, demonstrating that our stimulus sets were properly orthogonalised. LDA with GIST predicted shape above chance level, at $87.5 \%$ for Set A and $69 \%$ for Set B. Category was predicted below chance level, at 37.5\% for Set A and $10 \%$ for Set B.

Shape and category RSA on all CNN layers for stimulus sets A and B. Figure 2 illustrates layer-by-layer RSA between the CNN representations and the shape and category models in the two stimulus sets. Note that all applications of RSA using the Set A behavioural models involved partial correlations (see Results: Behavioural). Looking across all networks, in the first layer of all CNNs, shape is already represented above the significance threshold in most cases, whereas category is not. We determined the significance threshold of every correlation by comparing it with a null distribution obtained by randomly permuting the RDM labels and then calculating dissimilarity relationships 1000 times. Shape correlations at the first layer of CNNs are lower and closer to the significance threshold for Set A $(0.12<\rho<0.24)$ than Set B $(0.24<\rho<0.36)$. For Set A, shape correlations remain relatively high at the final layer $(0.29<\rho<0.59)$. In contrast, for Set $\mathrm{B}$, shape correlation levels increase in the networks before falling in the final layers of all networks, to roughly their initial values $(0.32<\rho<0.36)$. For all networks, category information remains low across the majority of layers, hovering at or below the significance level until the final few layers, where it increases above the significance threshold to peak at the final layer. At the final layer, for Set A, category correlations reach between $0.34<\rho<0.42$. For Set B, category correlations reach between $0.24<\rho<0.37$ at the final layer. The values of final layer correlations are presented in Table 1. Set B correlations with CaffeNet, VGG-19 and GoogLeNet are reproduced from Kubilius et al. ${ }^{18}$.

To investigate the interaction between shape and category and CNN layers, we tested correlation values in a $2 \times 2$ ANOVA with Layer (modelled linearly with intercept and slope) and Condition (Shape or Category) as factors. Table 2 summarises the statistical results of the main effects (Layer, Condition) and their interaction in CNNs and models. For Set A, across all networks, Layer has a highly significant main effect and Condition is also significant (Table 2) which suggests that correlation values can be predicted given the CNN layer and the condition of interest (shape or category information). Their interaction is significant in GoogleNet and VGG-19, but not in CaffeNet and ResNet50, suggesting that as category information increases, shape information decreases significantly in two out of the four networks tested. For Set B, across all networks, Condition is highly significant, and Layer has a significant main effect in behavioural model correlations. This suggests that it is possible to make significant predictions of behavioural shape and category judgements given CNN layer information. For Set B, Condition is highly significant across all networks, and the interaction between Layer and Condition is only significant in CaffeNet.

In summary, across both Sets A and B, we can see that shape information gradually increases and/or wavers as the network is traversed, before falling in the final layers. Across both Sets A and B, category information is at or below the significance threshold in the initial layer before reaching the maximum value at the final layer, showing the opposite trend with shape correlations. Figure 3 contains multidimensional scaling plots of peak shape and category information for Sets A and B.

Shape versus category information in visual ventral stream regions. Figure 4 summarises the representational similarity in three regions of interest (ROIs) along the visual ventral pathway, from low-level area V1 through to posterior and anterior VTC, compared with models of shape and category. Note that all RSA using Set A involved partial correlations (explained above in Results: Behavioural). For Set A, shape information reduces along the ventral stream, from $18 \%$ to $10 \%$. Category information increases along the ventral pathway, from $-6 \%$ to $40 \%$. We tested RSA results using a two-factor ANOVA, with ROI (V1, VTC ant, VTC post) and Condition (category, shape) as within-subject factors. For Set A, results reveal a significant main effect for ROI $\left(F_{2,15}=35.81, p<0.001\right)$, whereas the main effect of Condition (shape vs category) is not significant $\left(F_{1,15}=1.02\right)$. There is a significant interaction between ROI and Condition $\left(F_{2,15}=73.34, p<0.001\right)$, indicating that as category information increases from V1 to VTC ant, shape information decreases. Post hoc pairwise t-tests further confirmed the dissociation between shape and category along the visual ventral stream: category divisions were able to significantly better explain the neural pattern in later ventral areas (VTC ant) relative to shape $\left(t_{(15)}=5.67\right.$, $p<0.0001)$; whereas the opposite was true in early visual area $\mathrm{V} 1$, where shape was significantly more related to the neural data compared to category divisions $\left(t_{(15)}=8.16, p<0.0001\right)$.

For Set B, we see a qualitatively similar trend of decreasing shape information from V1 to VTC anterior (from $18 \%$ to $4 \%$ ) and increasing category information (from $1 \%$ to $6 \%$ ). The two-factor ANOVA, with ROI (V1, VTC ant, VTC post) and Condition (Category, Shape), revealed that when correlating ROI representations with the models for Set $\mathrm{B}$, the effect of ROI is significant $\left(F_{2,14}=3.79, p=0.027\right)$, as is Condition $\left(F_{1,14}=33.84, p<0.001\right)$ and there is a highly significant interaction effect between ROI and Condition $\left(F_{2,14}=13.33, p<0.001\right)$. Again, pairwise $t$-tests further confirmed the dissociation between shape and category in visual brain regions, with Shape being significantly more related to neural data in early visual area V1 than Category $\left(t_{(14)}=5.28, p=0.0001\right)$. Category was not able to significantly explain neural patterns more in VTC ant than shape $\left(t_{(14)}=1.20, p=0.24\right)$. Overall, these results illustrate a decrease in shape combined with an increase in category going from V1 to VTC anterior. 
A

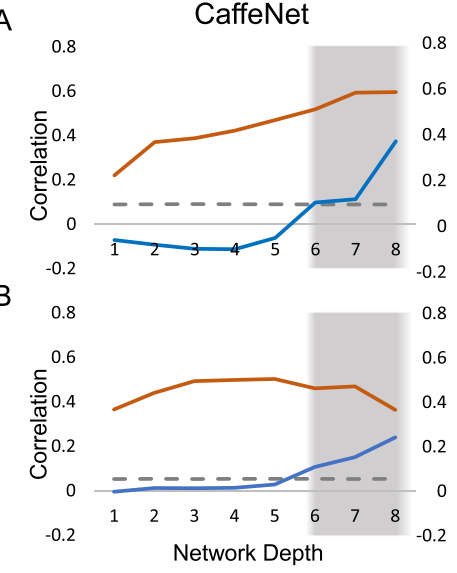

VGG-19

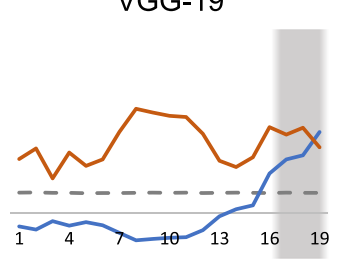

GoogLeNet
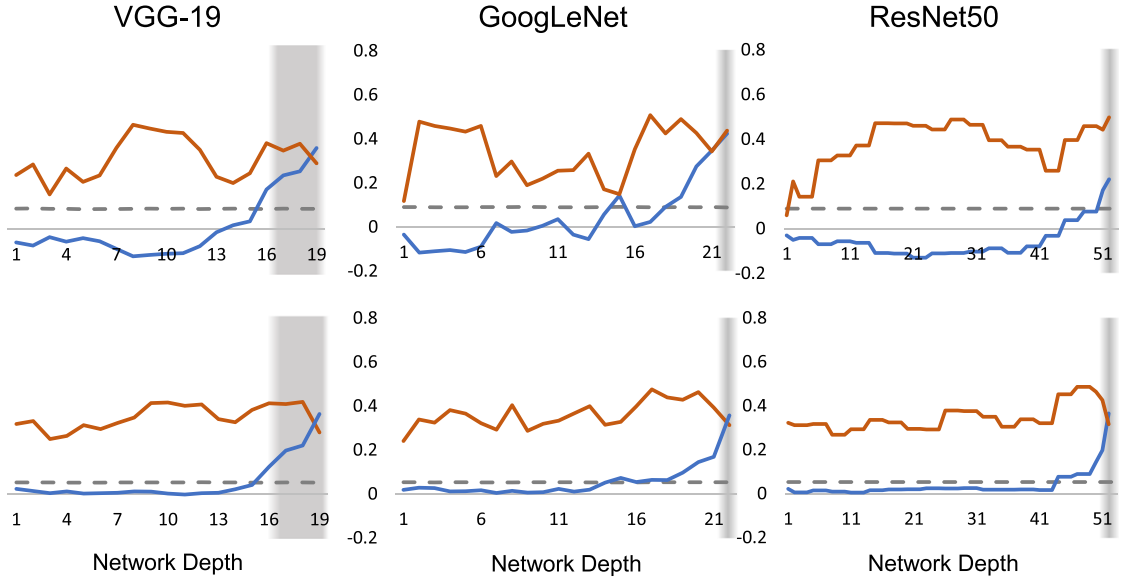

- Category - Shape

Figure 2. Correlation between layers in CNNs and shape (orange) versus category (blue) in Set A (top row) and $\mathrm{B}$ (bottom row). The horizontal axis indicates network depth and the vertical axis indicates correlation (Spearman's $\rho$ ). For GoogLeNet and ResNet architectures, the correlations shown are for $3 \times 3$ convolutional operations, while other parallel operations (projections and convolutions of different sizes) are omitted. Dashed line indicates significance threshold of $\mathrm{p}<0.05$, which was calculated by randomly permuting the RDM labels and then calculating dissimilarity relationships 1000 times. Grey shading indicates fully-connected layers. Results for Set B CaffeNet, VGG-19 and GoogLeNet were previously reported in Kubilius et al. ${ }^{18}$.

\begin{tabular}{|l|l|l|l|l|}
\hline \multirow{2}{*}{ Network } & \multicolumn{2}{|l|}{ Set A } & \multicolumn{2}{l|}{ Set B } \\
\cline { 2 - 5 } & Shape & Category & Shape & Category \\
\hline CaffeNet & $\mathbf{0 . 5 9 6 5}$ & 0.3737 & $\mathbf{0 . 3 6 3 9}$ & 0.2394 \\
\hline VGG-19 & 0.2888 & 0.3556 & 0.2786 & 0.3605 \\
\hline GoogLeNet & 0.4375 & $\mathbf{0 . 4 2 4 7}$ & 0.3125 & 0.3569 \\
\hline ResNet50 & 0.4978 & 0.2209 & 0.3159 & $\mathbf{0 . 3 6 5 2}$ \\
\hline
\end{tabular}

Table 1. Final network layer correlations with shape and category for Set A and B. Maximum values across the four networks are highlighted in bold.

\begin{tabular}{|l|l|l|l|l|l|l|l|l|}
\hline $\begin{array}{l}\text { Stimulus } \\
\text { Set }\end{array}$ & Network & $\begin{array}{l}\text { Number of } \\
\text { Layers }\end{array}$ & Layer $\boldsymbol{F}_{\boldsymbol{1}, \boldsymbol{1}}$ & Layer $\boldsymbol{p}$ & Condition $\boldsymbol{F}_{\boldsymbol{l}, 1}$ & Condition $\boldsymbol{p}$ & Interaction $\boldsymbol{F}_{\boldsymbol{l}, \boldsymbol{l}}$ & Interaction $\boldsymbol{p}$ \\
\hline \multirow{4}{*}{ A } & CaffeNet & 8 & 41.233 & $<0.001$ & 126.651 & $<0.001$ & 0.202 & 0.661 \\
\cline { 2 - 9 } & VGG-19 & 19 & 17.370 & $<0.001$ & 99.161 & $<0.001$ & 6.252 & 0.017 \\
\cline { 2 - 9 } & GoogLeNet & 22 & 18.59 & $<0.001$ & 87.68 & $<0.001$ & 10.21 & 0.003 \\
\cline { 2 - 9 } & ResNet50 & 52 & 25.010 & $<0.001$ & 750.551 & $<0.001$ & 0.323 & 0.571 \\
\hline \multirow{4}{*}{ B } & CaffeNet & 8 & 8.306 & 0.014 & 212.106 & $<0.001$ & 7.774 & 0.016 \\
\cline { 2 - 9 } & VGG-19 & 19 & 22.075 & $<0.001$ & 207.91 & $<0.001$ & 3.536 & 0.069 \\
\cline { 2 - 9 } & GoogLeNet & 22 & 27.727 & $<0.001$ & 329.938 & $<0.001$ & 1.833 & 0.183 \\
\cline { 2 - 8 } & ResNet50 & 52 & 61.007 & $<0.001$ & 1108.272 & $<0.001$ & 0.311 & 0.578 \\
\hline
\end{tabular}

Table 2. $2 \times 2$ ANOVA results of Layer (modelled linearly with slope and intercept) and Condition (shape or category) and their interaction in CNNs and models.

RSA for fMRI brain data and all CNN layers. Neural fMRI responses for each participant, and ROI, for Set A and Set B were correlated with the RDMs of every layer for each CNN. Results are shown in Fig. 5. For each stimulus set and network, correlation values were tested in a $2 \times 3$ ANOVA with Layer (modelled linearly with intercept and slope) and ROI as within subject factors. In CaffeNet, V1 and VTC posterior correlations peaked at the third convolutional layer, and VTC anterior peaks at the final layer for both stimulus sets. For both stimulus sets, the $2 \times 3$ ANOVA results reveal a significant main effect of ROI (Set A: $F_{2,15}=88.73, p<0.001$; Set B: $\left.F_{2,14}=57.00, p<0.001\right)$ and Layer (Set A: $F_{1,15}=41.06, p<0.001 ; F_{1,14}=48.38, p<0.001$ ) and their interaction (Set A: $F_{2,15}=133.72, p<0.001$; Set B: $F_{2,14}=44.88, p<0.001$ ). In VGG-19, both stimulus sets show similar peaks in correlations, with V1 reaching a maximum at layer 13, VTC posterior at layer 15, and VTC anterior at the final $19^{\text {th }}$ layer. For both sets, there is a significant main effect of ROI (Set A: $F_{2,15}=59.12, p<0.001$; Set B: $F_{2,14}=26.98, p<0.001$ ) and Layer (Set A: $F_{1,15}=294.14, p<0.001 ; F_{1,14}=40.30, p<0.001$ ). The ROI $\mathrm{x}$ Layer 
CaffeNet

A. 1

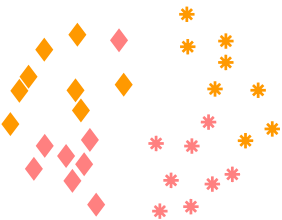

A.2

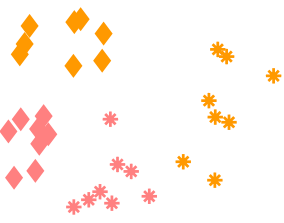

B.1
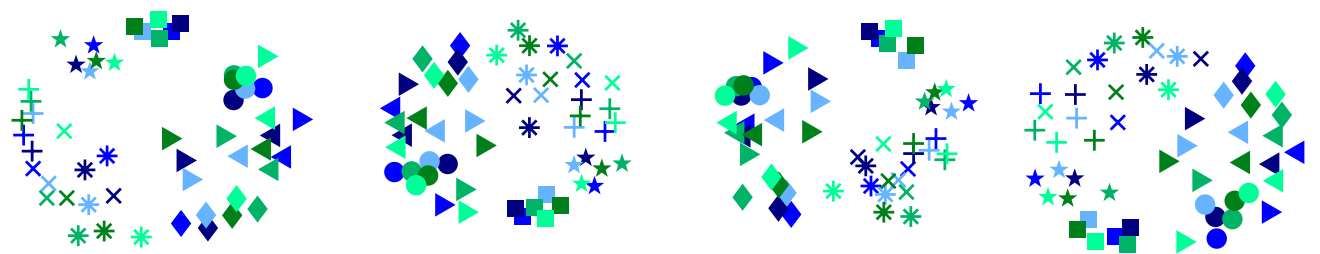

B.2
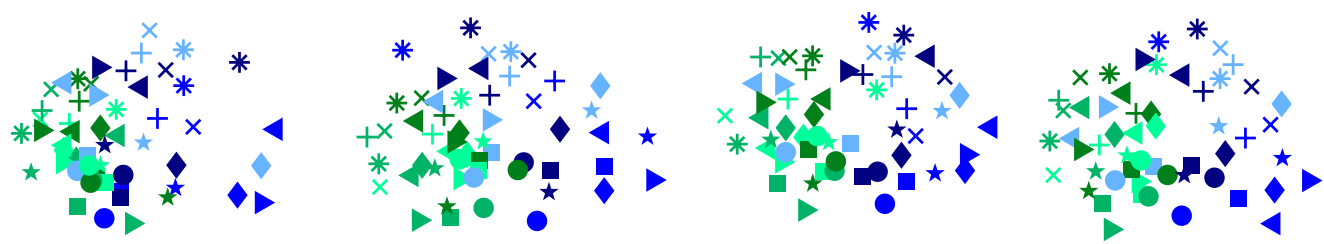

Figure 3. Multidimensional scaling plots of (1) Peak shape correlations with common shape represented by common symbols, and (2) peak category correlations, with common category represented by shared colour, for each network and Set A (top 2 rows) and B (bottom 2 rows). Colour coding corresponds to Fig. 1.

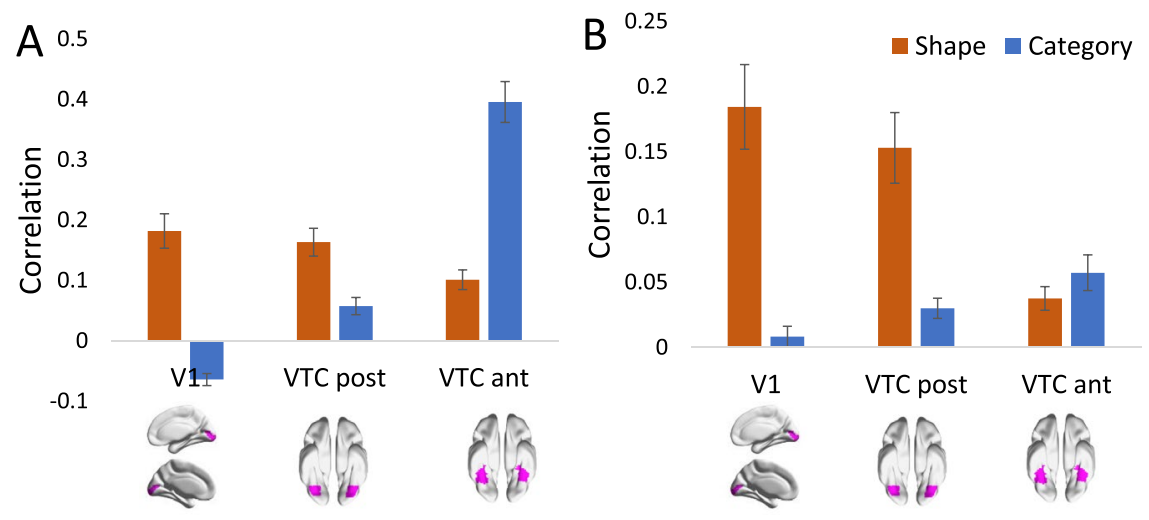

Figure 4. RSA results for shape and category models for Set A (left) and B (right) in ROIs. Three regions along the ventral visual pathway are analysed: V1, VTC post and VTC ant. Error bars represent standard error. ROI visualisations are re-used from (A) in (Bracci, Kalfas, \& Op de Beeck ${ }^{34}$, p. 8). Note the difference in scale between $\mathrm{A}$ and $\mathrm{B}$.

interaction is significant in Set $\mathrm{A}\left(F_{2,15}=55.49, p<0.001\right)$, but does not reach significance in Set $\mathrm{B}\left(F_{2,14}=2.76\right.$, $p=0.06)$. GoogLeNet has multiple peaks for correlations with V1 and VTC posterior, and there is a clear peak in VTC anterior in the final layer for both stimulus sets. For both Sets, ROI (Set A: $F_{2,15}=73.76, p<0.001$; Set B: $F_{2}$, ${ }_{14}=37.07, p<0.001$ ), Layer (Set A: $F_{1,15}=152.19, p<0.001$; Set B: $F_{1,14}=18.08, p<0.001$ ) and their interaction (Set A: $F_{2,15}=130.85, p<0.001$; Set B: $F_{2,14}=12.46, p<0.001$ ) are all highly significant. Finally, in ResNet50, V1 peaks at layers 44 to 47 , VTC posterior peaks at layers 47 to 49 , and VTC anterior peaks at the final layer. For 

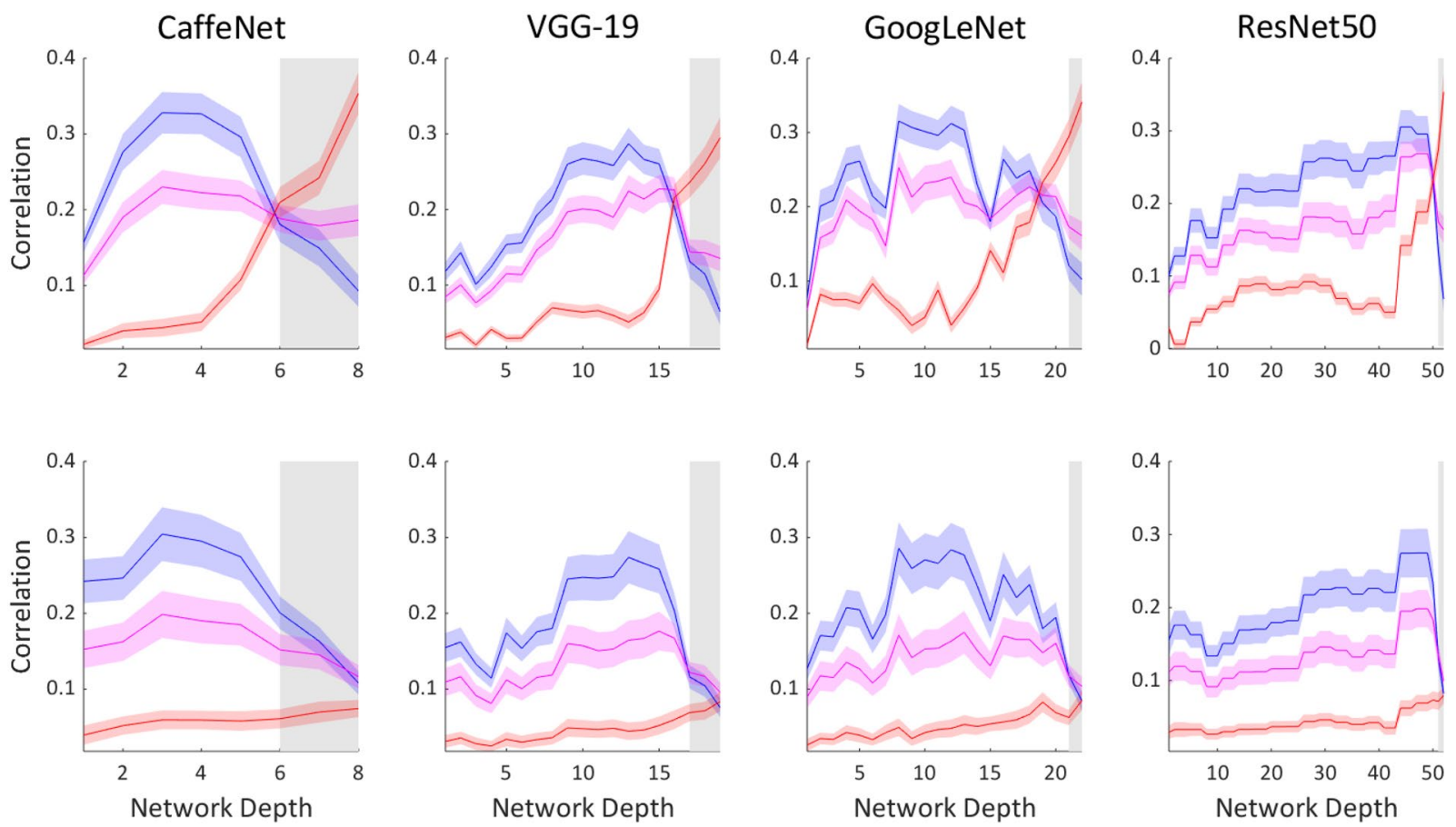

- V1 $=$ VTC post $=$ VTC ant

Figure 5. RSA comparing models (CaffeNet, VGG-19, GoogLeNet and ResNet50) and fMRI activation in V1 (blue), VTC post (magenta) and VTC ant (red) ROIs for Sets A (top row) and B (bottom row). Grey shading indicates fully-connected layers. Shading indicates SEM across subjects.

both Sets, ROI (Set A: $F_{2,15}=31.20, p<0.001$; Set B: $F_{2,14}=20.26, p<0.001$ ) and Layer (Set A: $F_{1,15}=1431.40$, $p<0.001$; Set B: $\left.F_{1,14}=895.32, p<0.001\right)$ are highly significant, and their interaction is significant (Set A: $F_{2}$, ${ }_{15}=5.97, p=0.003$; Set B: $\left.F_{2,14}=52.54, p<0.001\right)$. Together these results show that across all deep neural networks, there is a cascade in correlation peaks from V1 to VTC posterior to VTC anterior along the layers of each network, matching with the flow of activation along the human visual ventral pathway. For all networks, and both stimulus sets, the highest correlation of VTC anterior occurs at the final layer. Post hoc Tukey tests showed that all pair-wise comparisons between V1 and VTC anterior were significant for all four networks and both stimulus sets (all $\mathrm{p}<0.005)$. Paired t-tests show a significant increase between the first and final layers of CNNs correlated with VTC anterior for Set B for all networks (CaffeNet: $\mathrm{t}(13)=2.871, \mathrm{p}=0.013$; VGG-19: $\mathrm{t}(13)=4.105, \mathrm{p}=0.001$, GoogLeNet: $\mathrm{t}(13)=4.524, \mathrm{p}<0.001$, ResNet50: $\mathrm{t}(13)=4.095, \mathrm{p}=0.001)$. We also ran a one sample $\mathrm{t}$-test and found that across all networks, the correlations between the final four layers and the activation of VTC anterior across subjects were significantly different from zero, for both datasets (Set A: $p<0.0001$, Set $\mathrm{B}: p<=0.0006$ ).

Explained variance from shape versus category information in correlations between fMRI brain data and CNN layers. We partialled out shape and category when correlating between brain data and CNN layers to investigate the level of variance that could be explained by each of these factors individually. Figure 6 shows partial correlations for all CNNs and V1 (first two columns) and VTC anterior (last two columns), partialling out shape (magenta) or category (blue). Zero is indicated by the dotted line.

Looking at partial correlations with VTC anterior, for Set A, when shape is partialled out (magenta), the correlation values drop, which is expected given that the two dimensions are partially correlated. However, importantly, category alone (indicated by the blue line) in anterior VTC is still significant in the final 3 layers of all CNNs (CaffeNet: $\mathrm{t}(14)=3.306, \mathrm{p}=0.005, \mathrm{t}(14)=4.166, \mathrm{p}<0.0001, \mathrm{t}(14)=9.055, \mathrm{p}<0.0001$; VGG19: $\mathrm{t}(14)=7.608, \mathrm{p}<0.0001, \mathrm{t}(14)=7.620, \mathrm{p}<0.0001, \mathrm{t}(14)=7.758, \mathrm{p}<0.0001$; GoogLeNet: $\mathrm{t}(14)=7.015$,

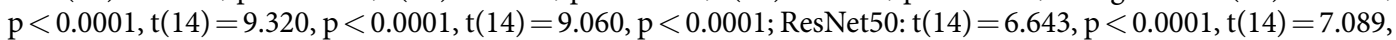
$\mathrm{p}<0.0001, \mathrm{t}(14)=10.033, \mathrm{p}<0.0001)$. This is visible and clearly consistent in all four CNNs. For Set $\mathrm{B}$, in anterior VTC, the drop in correlation values when partialling out shape is not apparent in any of the CNNs, confirming that the two dimensions are already independent in the stimulus set.

Looking at the first row of Fig. 6, we see that partial correlations between V1 and both datasets follow a similar pattern. When partialling out shape or category, correlations are still quite high along the convolutional layers, indicating that there is additional information contained in V1 and these CNN layers that is not accounted for by shape or category alone. Across all layers, the information contained by partialling out shape (magenta) is lower than the information contained by partialling out category (blue). The main decrease in these partial correlations is seen in the final fully connected layers, where there is near zero information related to category that is in 


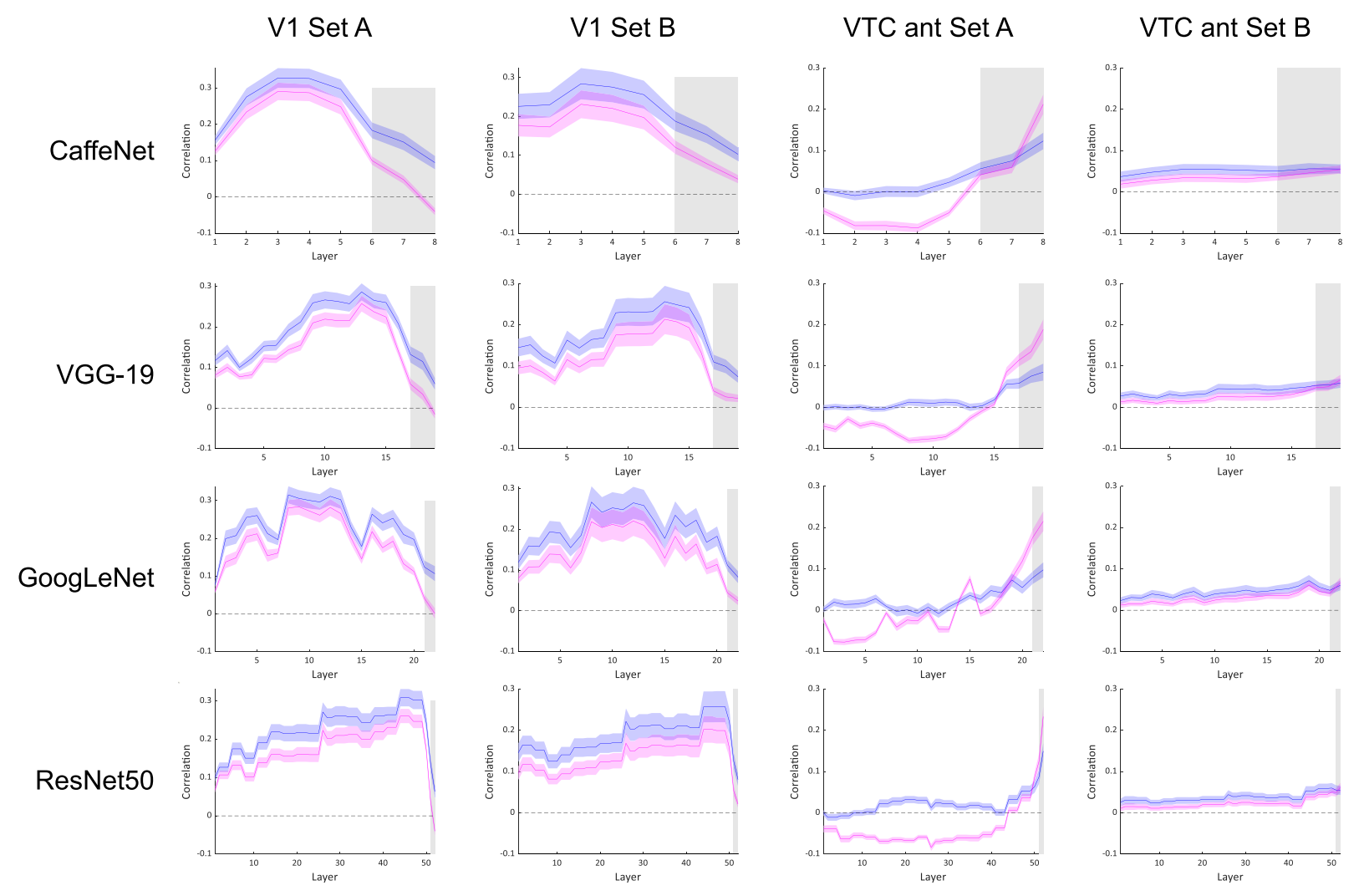

Figure 6. Partial correlations between CNNs and V1 (first two columns) or VTC ant (last two columns). Magenta shows shape information with category partialled out, blue shows category information with shape partialled out. Error bars show SEM across subjects.

common in $\mathrm{V} 1$ or in the final layers of CNNs. There is still some correlation related to shape (indicated by the blue line) that is preserved in V1 and in the final layers of CNNs.

\section{Discussion}

In this study, we investigated orthogonal shape and category representations in biological and artificial networks by making comparisons between: (i) CNNs and behavioural models of shape and category; (ii) behavioural models and the brain; and (iii) CNNs and the brain. First, comparing artificial networks and models, we found that CNNs represent category information as well as shape, and that category information peaks at the final layer for all tested CNNs, regardless of network depth. Second, comparing models and the brain, there is a two-way interaction between shape and category in the human visual ventral pathway, where shape is best represented earlier in V1, and category emerges later in anterior VTC. This interaction between shape and category is significant across both stimulus sets. Third, comparing artificial networks and the brain, V1 correlates highest with early to mid-level layers of deep networks, and anterior VTC correlates best with the final layer of CNNs. Across both stimulus sets and for all networks, peak correlations with V1 always occur in earlier network layers than peak correlations with anterior VTC, demonstrating that CNNs reflect a similar order of computational stages as the human ventral pathway when processing these object images.

Our results allow for a greater understanding of how shape and category are represented in deep networks and in the visual ventral pathway, in particular: (i) how differing shape and category definitions between the two stimulus sets reveal differences between low-level and high-level shape representations in CNNs and the brain; (ii) how shape and category processing along deep network layers maps onto brain regions; and iii) how careful stimulus design allows us to make better inferences about category information in the brain and in CNNs.

One major advantage of this study is that we consider two stimulus sets that carefully control shape and category to draw conclusions about their interaction and interplay, rather than broadly extrapolating results based on a single set of images. These two well-controlled stimulus sets are similar in design but differ slightly in how shape and category are defined, allowing us to extract a finer interpretation of results. Looking at the differences in shape definitions between these stimulus sets, in Set A, shape is defined with a low to high aspect ratio (described as "bar-like" or "blob-like"), while it is characterized retinotopically in Set B. Comparing CNNs and models, both low-level (Set B) and high-level (Set A) shape information is preserved until the very last layer of all networks, however there is a visible reduction in low-level compared to high-level shape information in the final layers. Comparing models and the brain, we see that the high-level (Set A) shape information remains quite high in VTC ant, compared to low-level (Set B) shape information, which reduces to correlation levels that are at or near zero. The plausible explanation for why shape information drops off in Set B but not in A, is 
that higher level regions represent a more abstract form of shape, which is factored into the design of Set A, but not B. Indeed, previous studies showed that perceived shape similarity strongly overlaps with higher-level brain representations in humans ${ }^{41}$, and in monkeys ${ }^{12,42}$. Kalfas et al. ${ }^{12}$ found that the deepest layers of networks, rather than IT responses, correlated best with human shape similarity judgements. We also found that CNNs correlated much higher with behavioural shape judgements than fMRI. This finding suggests that there is at least some correspondence between how humans and models use shape, even though there are very likely also differences (see e.g. Baker et al. ${ }^{19}$ and Geirhos et al. ${ }^{21}$ ).

Considering the differences in category definitions between the stimulus sets, Set A has only two category clusters defined by the animate-inanimate division, whereas Set B has six object clusters. The number of groups clearly affects the size difference in correlation levels between category models and CNNs as well as the brain, where fewer groupings boost the signal. In the final layer of all CNNs, we see that category, as defined by animacy in Set A, reaches correlation levels up to three times the magnitude of Set B. Considering brain data, category as defined by animacy in Set A reaches six times the magnitude in VTC ant compared to Set B. However, when the contributions of category and shape are analysed separately, the difference between Sets A and B, in terms of category information, disappears. This is evident in Fig. 6, with the blue line showing a very similar peak in both datasets for all DNNs. The very high correlations for category that were observed in Fig. 5 for Set A were mainly driven by the shape component, and when this is dissociated the results across the two stimuli sets largely correspond (Fig. 6). We find that in all four networks, human similarity judgements of category are best explained by the final layer of CNNs, more so than fMRI representations in late ventral areas.

It may seem striking that in some cases the correlations for shape remain higher than those for category, even at the final layer (see Fig. 2, CaffeNet both sets and ResNet50 Set A). The shape model itself is a behavioural model, which captures high-level properties of shape that are presumably, still represented at the final layers of CNNs. If the shape model was defined instead using basic low-level shape features, we would expect more of a decrease in the final layers of CNNs.

Our use of multiple CNNs allows us to observe the influence of network depth on peak correlations with brain regions. Hong et al. ${ }^{9}$ compared their brain data to a CNN consisting of 6 parallelised convolutional layers, finding that the model's top hidden layer was most predictive of IT response patterns and that lower layers had higher resemblance to V1-like Gabor patterns. Consistent with their findings, we also found that the final layer of CNNs had maximum correspondence with later ventral stream areas, and that earlier layers showed higher correlation with V1. Cichy et al. ${ }^{14}$ found peak V1 correlations in the second layer of an 8-layer CNN trained for object recognition. Similarly in our experiments, we found that peak V1 correlations occurred at layer 3 in an 8-layer network (CaffeNet) for both stimulus sets. As network depth increases, peak correlations with V1 shift from earlier tiers in the network to later layers. Interestingly, some of the highest V1 correlations occur immediately prior to fully connected layers, as is the case in ResNet50 and VGG-19.

Figure 5 illustrates peak V1 correlations occurring as late as the $45^{\text {th }}$ layer in ResNet50, bringing into question the explanatory value of additional processing stages in deeper networks, especially when an 8-layer network achieves similar magnitudes of correlation with V1 by the third layer. Nevertheless, while the maximum correlation values of brain regions shift to later layers in larger networks, the rank-order of correlation peaks with brain regions still matches the order of information processing along the ventral pathway. That is, correlations with V1 always peak before VTC ant, regardless of network depth. We extend upon the findings of Cichy et al. ${ }^{14}$ that early visual area correlations peak before later ones, from a single 8 layer network to multiple networks, including a 50 layer network.

In conclusion, despite shape and category often being confounded in natural images, and the possibility for artificial neural networks to exploit this correlation when performing classification tasks, we find that deep convolutional neural networks are able to represent category information independently from low-level shape in a manner similar to higher level visual cortex in humans.

\section{Data availability}

The datasets generated during and/or analysed during the current study are available from the corresponding author on reasonable request.

Received: 24 January 2019; Accepted: 22 January 2020;

Published online: 12 February 2020

\section{References}

1. Krizhevsky, A., Sutskever, I., \& Hinton, G. E. ImageNet Classification with Deep Convolutional Neural Networks. Advances in Neural Information Processing Systems 25 (NIPS 2012), pp. 1097-1105. Lake Tahoe: Curran Associates, Inc. (2012).

2. Szegedy, C. et al. Going deeper with convolutions. 2015 IEEE Conference on Computer Vision and Pattern Recognition (CVPR), pp. 1-9. Boston, MA (2015).

3. Simonyan, K., \& Zisserman, A. Very Deep Convolutional Networks for Large-Scale Image Recognition. ICLR, (2015).

4. He, K., Zhang, X., Ren, S., \& Sun, J. Delving Deep into Rectifiers: Surpassing Human-Level Performance on ImageNet Classification. 2015 IEEE International Conference on Computer Vision (ICCV), pp. 1026-1034. Santiago (2015).

5. Kriegeskorte, N. Deep Neural Networks: A New Framework for Modeling Biological Vision and Brain Information Processing. Annual Review of Vision Science 1, 417-446, https://doi.org/10.1146/annurev-vision-082114-035447 (2015).

6. Kietzmann, T. C., McClure, P., \& Kriegeskorte, N. Deep Neural Networks In Computational Neuroscience. bioRxiv. Preprint at, https://doi.org/10.1101/133504 (2017).

7. Khaligh-Razavi, S.-M. \& Kriegeskorte, N. Deep Supervised, but Not Unsupervised, Models May Explain IT Cortical Representation. PLoS Computational Biology, 10(11), e1003915, https://doi.org/10.1371/journal.pcbi.1003915 (2014).

8. Cadieu, C. F. et al. Deep Neural Networks Rival the Representation of Primate IT Cortex for Core Visual Object Recognition. PLos Computational Biology 10(12), e1003963, https://doi.org/10.1371/journal.pcbi.1003963 (2014). 
9. Yamins, D. L., et al. Performance-optimized hierarchical models predict neural responses in higher visual cortex. (Sejnowski, T. J., Ed.) PNAS, 111(23), 8619-8624, https://doi.org/10.1073/pnas.1403112111 (2014).

10. Hong, H., Yamins, D. L., Majaj, N. J. \& DiCarlo, J. J. Explicit information for category-orthogonal object properties increases along the ventral stream. Nature Neuroscience 19(4), 613-622, https://doi.org/10.1038/nn.4247 (2016).

11. Güçlü, U. \& van Gerven, M. A. Deep Neural Networks Reveal a Gradient in the Complexity of Neural Representations across the Ventral Stream. The Journal of Neuroscience 35(27), 10005-10014 (2015).

12. Kalfas, I., Vinken, K. \& Vogels, R. Representations of regular and irregular shapes by deep Convolutional Neural Networks, monkey inferotemporal neurons and human judgments. PLoS Computational Biology 14(10), e1006557, https://doi.org/10.1371/journal. pcbi.1006557 (2018).

13. Devereaux, B. J., Clarke, A. \& Tyler, L. K. Integrated deep visual and semantic attractor neural networks predict fMRI patterninformation along the ventral object processing pathway. Scientific Reports 8, 10636, https://doi.org/10.1038/s41598-018-28865-1 (2018).

14. Cichy, R. M., Khosla, A., Pantazis, D., Torralba, A. \& Oliva, A. Comparison of deep neural networks to spatio-temporal cortical dynamics of human visual object recognition reveals hierarchical correspondence. Scientific Reports 6, 27755, https://doi. org/10.1038/srep27755 (2016).

15. Bracci, S., Ritchie, J. B. \& Op de Beeck, H. On the partnership between neural representations of object categories and visual features in the ventral visual pathway. Neuropsychologia 105, 153-164 (2017).

16. Bracci, S. \& Op de Beeck, H. Dissociations and Associations between Shape and Category. The Journal of Neuroscience 36(2), 432-444 (2016).

17. Belongie, S., Malik, J. \& Puzicha, J. Shape Matching and Object Recognition Using Shape Contexts. IEEE Transactions on Pattern Analysis and Machine Intelligence 24(4), 509-522 (2002).

18. Kubilius, J., Bracci, S. \& Op de Beeck, H. P. Deep Neural Networks as a Computational Model for Human Shape Sensitivity. PLoS Computational Biology 12(4), e1004896, https://doi.org/10.1371/journal.pcbi.1004896 (2016).

19. Baker, N., Lu, H., Erlikhman, G. \& Kellman, P. Deep convolutional networks do not classify based on global object shape. PLoS Computational Biology 14(12), e1006613, https://doi.org/10.1371/journal.pcbi.1006613 (2018).

20. Ritter, S., Barrett, D. G., Santoro, A., \& Botvinick, M. M. Cognitive Psychology for Deep Neural Networks: A Shape Bias Case Study. Proceedings of the 34th International Conference on Machine Learning, PMLR 70, pp. 2940-2949. Sydney, Australia (2017).

21. Geirhos, R. et al ImageNet-trained CNNs are biased towards texture; increasing shape bias improves accuracy and robustness. arXiv, 1811.12231. Retrieved from, https://arxiv.org/abs/1811.12231 (2018).

22. Kaiser, D., Azzalini, D. C. \& Peelen, M. V. Shape-independent object category responses revealed by MEG and fMRI decoding. Journal of Neurophysiology 115, 2246-2250, https://doi.org/10.1152/jn.01074.2015 (2016).

23. Proklova, D., Kaiser, D. \& Peelen, M. V. Disentangling Representations of Object Shape and Object Category in Human Visual Cortex: The Animate-Inanimate Distinction. Journal of Cognitive Neuroscience 28(5), 680-692 (2016).

24. Grill-Spector, K. \& Weiner, K. S. The functional architecture of the ventral temporal cortex and its role in categorization. Nature Reviews Neuroscience 15(8), 536-548, https://doi.org/10.1038/nrn3747 (2014).

25. Kriegeskorte, N. et al. Matching Categorical Object Representations in Inferior Temporal Cortex of Man and Monkey. Neuron 60(6), 1126-41, https://doi.org/10.1016/j.neuron.2008.10.043 (2008).

26. Kiani, R., Esteky, H., Mirpour, K. \& Tanaka, K. Object category structure in response patterns of neuronal population in monkey inferior temporal cortex. Journal of Neurophysiology 97, 4296-4309 (2007).

27. Rice, G. E., Watson, D. M., Hartley, T. \& Andrews, T. J. Low-Level Image Properties of Visual Objects Predict Patterns of Neural Response across Category-Selective Regions of the Ventral Visual Pathway. Journal of Neuroscience 34(26), 8837-8844, https://doi. org/10.1523/JNEUROSCI.5265-13.2014 (2014).

28. Andrews, T. J., Watson, D. M., Rice, G. E. \& Hartley, T. Low-level properties of natural images predict topographic patterns of neural response in the ventral visual pathway. Journal of Vision 15(7), 1-12, https://doi.org/10.1167/15.7.3 (2015).

29. Baldassi, C. et al. Shape Similarity, Better than Semantic Membership, Accounts for the Structure of Visual Object Representations in a Population of Monkey Inferotemporal Neurons. PLoS Computational Biology 9(8), e1003167, https://doi.org/10.1371/journal. pcbi.1003167 (2013).

30. Ritchie, J. B. \& Op de Beeck, H. Using neural distance to predict reaction time for categorizing the animacy, shape, and abstract properties of objects. Scientific Reports 9, 13201, https://doi.org/10.1038/s41598-019-49732-7 (2019).

31. Oliva, A. \& Torralba, A. Modeling the shape of the scene: a holistic representation of the spatial envelope. International Journal of Computer Vision 42(3), 145-175 (2001).

32. Kriegeskorte, N., Mur, M. \& Inverse, M. D. S. inferring dissimilarity structure from multiple item arrangements. Frontiers in Psychology 3, 245, https://doi.org/10.3389/fpsyg.2012.00245 (2012).

33. Op de Beeck, H. P. Against hyperacuity in brain reading: spatial smoothing does not hurt multivariate fMRI analyses? Neuroimage 49, 1943-1948 (2010).

34. Bracci, S., Kalfas, I., \& Op de Beeck, H. The ventral visual pathway represents animal appearance over animacy, unlike human behavior and deep neural networks. bioRxiv. Preprint at: https://doi.org/10.1101/228932 (2017)

35. Russakovsky, O. et al. ImageNet Large Scale Visual Recognition Challenge. International Journal of Computer Vision (IJCV), 115(3), 211-252, https://doi.org/10.1007/s11263-015-0816-y (2015).

36. Jia, Y. et al. Caffe: Convolutional Architecture for Fast Feature Embedding. Preprint at https://arxiv.org/abs/1408.5093 (2014).

37. He, K., Zhang, X., Ren, S., \& Sun, J. Deep Residual Learning for Image Recognition. ArXiv:1512.03385 [Cs]. Preprint at, http://arxiv. org/abs/1512.03385 (2015).

38. Greff, K., Srivastava, R. K., \& Schmidhuber, J. Highway and Residual Networks learn Unrolled Iterative Estimation. International Conference on Learning Representations (ICLR), (2017).

39. Kriegeskorte, N., Mur, M., \& Bandettini, P. Representational similarity analysis - connecting the branches of systems neuroscience. Frontiers in Systems Neuroscience, 2(4), https://doi.org/10.3389/neuro.06.004.2008 (2008).

40. Shepard, R. N. \& Chipman, S. Second-order isomorphism of internal representations: Shapes of states. Cognitive Psychology 1(1), 1-17 (1970).

41. Op de Beeck, H. P., Torfs, K. \& Wagemans, J. Perceived shape similarity among unfamiliar objects and the organization of the human object vision pathway. J. Neurosci. 28(40), 10111-10123, https://doi.org/10.1523/JNEUROSCI.2511-08.2008 (2008).

42. Op de Beeck, H., Wagemans, J. \& Vogels, R. Inferotemporal neurons represent low-dimensional configurations of parameterized shapes. Nature Neuroscience 4(12), 1244-1252 (2001).

\section{Acknowledgements}

A.A.Z. and H.O.d.B. were funded by grant C14/16/031 of the KULeuven Research Council. J.B.R. received funding from the FWO and European Union's Horizon 2020 research and innovation programme under the Marie Skłodowska-Curie grant agreement No. 665501, via a FWO [PEGASUS] ${ }^{2}$ Marie Skłodowska-Curie fellowship (12T9217N). S.B. is funded by FWO (Fonds Wetenschappelijk Onderzoek) postdoctoral fellowship $516(12 S 1317 N)$. Neuroimaging was funded by the Flemish Government Hercules Grant ZW11_10. We would like to thank Ginette Lafit and Tim Leers for help with statistical data analysis. 


\section{Author contributions}

All authors contributed to the study design. S.B., J.B.R. and H.O.d.B. provided pre-processed neuroimaging data and collected behavioural data. A.A.Z. ran network simulations, analysed the data and wrote the manuscript with input from all authors. All authors interpreted the data, edited the manuscript and approved the final version.

\section{Competing interests}

The authors declare no competing interests.

\section{Additional information}

Correspondence and requests for materials should be addressed to A.A.Z.

Reprints and permissions information is available at www.nature.com/reprints.

Publisher's note Springer Nature remains neutral with regard to jurisdictional claims in published maps and institutional affiliations.

Open Access This article is licensed under a Creative Commons Attribution 4.0 International License, which permits use, sharing, adaptation, distribution and reproduction in any medium or format, as long as you give appropriate credit to the original author(s) and the source, provide a link to the Creative Commons license, and indicate if changes were made. The images or other third party material in this article are included in the article's Creative Commons license, unless indicated otherwise in a credit line to the material. If material is not included in the article's Creative Commons license and your intended use is not permitted by statutory regulation or exceeds the permitted use, you will need to obtain permission directly from the copyright holder. To view a copy of this license, visit http://creativecommons.org/licenses/by/4.0/.

(C) The Author(s) 2020 